SIR, - The report of a stone fish "bite" by Drs T Llewellyn and A Fraser-Moodie' illustrates the result of having been "bitten" by a stone fish and makes recommendations for treatment.

The stone fish, genus synancejidae, does not bite but injects its venom by way of an adapted dorsal $\mathrm{fin}^{2}$ into the flesh of unwary bathers. The advice given that "the affected part should be immersed in hot water at up to $50^{\circ} \mathrm{C}$ to denature the venom" is a dangerous suggestion, as not only the venom but also the affected part will be denatured.

Moritz and Henriques showed that water at $47^{\circ} \mathrm{C}$ caused full thickness burns in humans after 18 minutes and that the process was without discomfort in some cases; water at $50^{\circ} \mathrm{C}$ caused full thickness burns after five minutes. ${ }^{3}$ Between $44^{\circ} \mathrm{C}$ and $51^{\circ} \mathrm{C}$ the time required to produce irreversible tissue damage is halved for each one degree rise in temperature (from 180 minutes to under two minutes). We think that the advice of Drs Llewellyn and Fraser-Moodie, although given for treatment of a potentially fatal injury, is incorrect and could well result in serious tissue destruction.

D J GORDON

R MILLAR

Burns Unit,

Royal Victoria Hospital

Belfast B'T12 6BA

1 Llewellyn T, Fraser-Moodie A. Stone fish bite. Br Med f 1990;300:134. (13 January.)

2 Anonymous. Encyclopaedia Britannica. 15th ed. Chicago: Encyclopaedia Britannica, 1988 .

3 Moritz AR, Henriques FC. Studies of thermal injury 11. Am f Pathol 1947;23:695-720.

\section{Single dose prophylaxis in colonic surgery}

SIR, - What a pity that the large, potentially valuable trial by Mr D C Rowe-Jones and others' should have been compromised by the need to obtain funding from a pharmaceutical company.

Many hospitals use the standard three dose regimen of cefuroxime plus metronidazole for prophylaxis against wound infection after colorectal surgery. The obvious question is whether a single dose would be as effective. Why complicate matters by substituting the more expensive cefotaxime for cefuroxime? The authors' explanation that they restricted the protocol to the two prophylactic regimens of principal interest sounds lame. Whose interest? Perhaps that of the manufacturers of cefotaxime, who presumably funded the trial.

The authors' intention to reduce costs is admirable, but they could have achieved an even greater saving if a single dose of cefuroxime plus metronidazole had been shown to be as good as three doses. If one of the principal aims of trials such as this is to reduce NHS costs then perhaps the NHS should fund them. Only then can we run the trials that we would like to see performed, rather than those the pharmaceutical companies are willing to finance.

JONATHAN D BEARD

Department of Surgery,

Leicester Roval Infirmary

Leicester LE2 7LX

1 Rowe-Jones DC, Peel ALG, Kingston RD, Shaw JFL, Teasdale C, Cole DS. Single dose cefotaxime plus metronidazole versus three dose cefuroxime plus metronidazole as prophylaxis against wound infection in colorectal surgery: multicentre prospective randomised study. $B r$ Med $\mathcal{F}$ 1990;300:18-22. (6 January.

AUTHOR'S REPLY, - I cannot accept that clinical research sponsored by a pharmaceutical company is by definition "compromised." It scarcely needs me to point out that a large proportion of clinical trials are funded directly or indirectly by the pharmaceutical industry as part of clinical development programmes or as academic research. The fact that such work is industry funded does not imply that its quality is necessarily to be questioned. Indeed, the skill of medical departments in the pharmaceutical industry in coordinating large, multicentre trials, supported by professional statistical advice and electronic data processing capacity, can be expected to enhance, rather than compromise, the quality of work done.

It is naive to imagine in the present financial climate that the NHS would fund antibiotic trials or that it is necessarily right that it should do so. In district general hospitals it is unusual to experience the luxury of research assistants and the like to help run clinical trials and it seems entirely appropriate to seek support from the pharmaceutical industry when, as in our case, the design and objective of the trial were determined by the principal clinicians concerned.

Our trial has shown that a single dose of cefotaxime and metronidazole is as effective for prophylaxis against wound infection after colorectal surgery as the standard three dose regimen of cefuroxime and metronidazole. I accept that it now remains to be shown which single dose regimen is the most effective; such a trial would need to be performed with the same high degree of statistical power as ours to allow comparison of the results with those we obtained and to avoid type II error.

Finally, I agree that cost is important but we deliberately avoided cost comparisons because of variations in hospital contract prices for antibiotics. Nevertheless, the current issue of $M I M S$ shows an equivalent dose of cefotaxime $(1 \mathrm{~g})$ to be less expensive than that of cefuroxime $(1.5 \mathrm{~g})$.

D C ROWE-JONES

Poole General Hospital

Poole BH15 2JB

SIR, - Mr D C Rowe-Jones and others imply that the improved pharmacokinetic profile of cefotaxime as a single preoperative dose explains its equal efficiency to three doses of cefuroxime in preventing wound infection after colorectal surgery.' The alternative explanation is that both antibiotics are equally effective but that the doses of cefuroxime given at the eighth and sixteenth hours after operation have no effect and are unnecessary. The experimental work by Burke would support the latter explanation.?

K T FISHWICK

R S CROTON

Surgical Unit,

Warrington District General Hospital,

Warrington WA5 $1 Q$ QG

1 Rowe-Jones DC, Peel ALG, Kingston RD, Shaw JFL, Teasdale $C$, Cole DS. Single dose cefotaxime plus metronidazole versus three dose cefuroxime plus metronidazole as prophylaxis three dose cefuroxime plus metronidazole as prophylaxis prospective randomised study. Br Med f 1990;300:18-22. prospective
(6 January.)

Burke JF. The effective period of preventive antibiotic action in experimental incision and dermal lesions. Surgery 1961;50: $161-8$

\section{Ovarian failure after total body irradiation}

SIR, - The article by Dr M P Cust and colleagues on ovarian failure after total body irradiation' prompts us to report a successful pregnancy after this treatment.

A 19 year old woman with chronic myeloid leukaemia received an allogeneic bone marrow transplant from a matched related donor after conditioning with high doses of cyclophosphamide and $7.5 \mathrm{~Gy}$ of unfractionated total body irradiation in March 1983. She achieved a complete haematological remission and in 1989 delivered a healthy baby after a normal pregnancy.

This is unusual but not unique. In 1988 Sanders et al reported the results on ovarian function in 187 women after bone marrow transplantation. ' In 144 patients with leukaemia treated with total body irradiation $(9 \cdot 2-10 \cdot 0$ Gy unfractionated or $12 \cdot 0-15 \cdot 8$ Gy fractionated over seven days) amenorrhoea was present for the first three years after transplantation. Subsequently, nine women, all aged less than 25 years at transplantation, had recovery of ovarian function between three and seven years (median four years) after transplantation. Three pregnancies were reported: one was terminated electively and the other two resulted in spontaneous abortion. Pregnancy after bone marrow transplantation conditioning with cyclophosphamide alone is not uncommon, ${ }^{2}$ and in younger women $(<25$ years) recovery of ovarian function is to be expected.

Our patient was the first bone marrow transplant recipient in Cardiff and received a lower dose total body irradiation than that used in most centres; we currently use $12.5 \mathrm{~Gy}$ fractionated irradiation. Nevertheless, recovery of ovarian function and successful pregnancy can occur after total body irradiation.

We have also seen many women who have developed psychosexual problems after bone marrow transplantation and we strongly support the need for counselling and advice on hormone replacement therapy after this treatment.

PETER M CUMBER J A WHITTAKER

University Hospital of Wales,

Cardiff CF+ $4 \mathrm{XN}$

1 Cust MP, Whitehead MI, Powles R, Hunter M, Milliken S Consequences and treatment of ovarian failure after total bod irradiation for leukaemia. Br Med 7 1989:299:1494-7. (16 December

2 Sanders JE, Buckner CD, Amos E, et al. Ovarian function following marrow transplantation for asplastic anaemia or leukaemia. $\mathcal{F}$ Clin Oncol 1988;6:813-8

3 Schmidt H, Ehninger G, Dopfer R, Waller HD. Pregnancy after bone marrow transplantation for severe aplastic anacmia. Bone Marrow Transplantation 1987;2:329-32.

\section{Organs for transplantation}

SIR, - We thank correspondents for many interesting observations on the confidential audit of all deaths in intensive care units, for which results for phase 2 of the study (1 April-30 June 1989) closely parallel those for phase 1 (1 January31 March 1989).' This was what we expected from the narrow confidence intervals in our first report.

Dr J N Payne and colleagues ${ }^{2}$ ask about data validation. In all, $30 \%$ of audit forms in phase 1 and $20 \%$ in phase 2 failed logical checks and were returned to intensive care units for resolution of queries. The checking program also identifies when the audit serial number is out of sequence with date of death and the unit concerned is asked to verify that all deaths have been reported. This check does not work as well as intended, however, because many intensive care units complete audit forms in order of admission date rather than date of death. Because the admission date is not recorded on the audit form no programatic check is possible. External checks through patient information systems - as reported in Trent-or by required referral of all people who die in intensive care units to transplantation coordinators-as practised in South East Thames Regional Health Authorityare to be encouraged. Regional liaison officers have responsibility for identifying the number of deaths in all intensive care units in their authority; an indirect check on the completeness of identification comes from interregional comparison of numbers of reported deaths in intensive care units per million residents. The high number of such deaths in South East Thames region could reflect comprehensive reporting.

The third audit report for deaths up to 31 December 1989 (phase 3) will analyse regional 